\title{
Tecnologias da Informação: a gestão de processos através de aplicativos de mensagem
}

\section{Information technologies: process management through message applications}

\author{
Paulo George Miranda Martins ${ }^{1}$, Dener Cesar Ferreira Lopes ${ }^{2}$, Rogério Aparecido Sá Ramalho ${ }^{3}$ \\ ${ }^{1}$ Mestrado pelo Programa de Pós-Graduação em Ciência da Informação pela UFSCar. ORCID: https://orcid.org/0000-0002-3922-5069 \\ ${ }^{2}$ Mestrando do Programa de Pós-Graduação em Ciência da Informação da UFSCar. ORCID: https://orcid.org/0000-0002-7967-6454 \\ ${ }^{3}$ Docente do Programa de Pós-Graduação em Ciência da Informação da UFSCar. ORCID: https://orcid.org/0000-0002-8491-3514
}

Autor para correspondência/Mail to: Paulo George Miranda Martins, pgeorgemm22@gmail.com

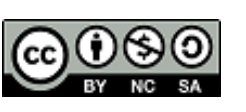

Copyright (C) 2019 Martins, Ramalho \& Lopes. Todo o conteúdo da Revista (incluindo-se instruções, política editorial e modelos) está sob uma licença Creative Commons Atribuição-NãoComercial-Compartilhalgual 3.0 Não Adaptada. Ao serem publicados por esta Revista, os artigos são de livre uso em ambientes educacionais, de pesquisa e não comerciais, com atribuição de autoria obrigatória. Mais informações em http://revistas.ufpr.br/atoz/about/submissions\#copyrightNotice.

\begin{abstract}
Resumo
Introdução: A partir do desenvolvimento das tecnologias de informação e comunicação, novas possibilidades de compartilhamento de dados e informações favoreceram mudanças significativas no comportamento da sociedade, contribuindo de forma acelerada para a globalização do conhecimento. No âmbito da manutenção de aeronaves, o emprego de tais tecnologias na gestão de processos produtivos ganha novos contornos a partir do uso de tecnologias capazes de prover maior controle de recursos, garantindo a confiabilidade dos serviços prestados e 0 controle dos gastos operacionais. O objetivo central desse trabalho é analisar as potencialidades da utilização de um aplicativo de mensagens na gestão de atendimentos em uma empresa de manutenção de aeronaves. Método: o procedimento metodológico adotado caracteriza-se pela pesquisa-ação, tendo como foco a temática de tecnologias da informação e comunicação aplicadas na gestão de processos. O que favorece o desenvolvimento de um modelo de gestão eficiente na distribuição de recursos empregados nas atividades de atendimento de tarefas. Resultados: como resultados descreve-se a relevância do uso do aplicativo de mensagens, pelo Controle da Qualidade, ao estabelecer um padrão no atendimento das demandas no contexto da manutenção de aeronaves, proporcionando melhorias e contribuindo para uma melhor visibilidade no detalhamento das tarefas e uma gestão mais eficiente dos recursos empregados nas solicitações realizadas pela manutenção. Conclusão: verifica-se que a aplicação de tais tecnologias proporciona uma maior sinergia entre os diversos setores envolvidos no processo, melhorando a comunicação e o direcionamento preciso dos recursos a serem empregados.
\end{abstract}

Palavras-chave: Tecnologias de informação e comunicação; Manutenção de aeronaves; Gestão de processos; Aplicativos de mensagem.

\begin{abstract}
Introduction: Considering the development of information and communication technologies, new possibilities for sharing data and information allowed significant changes in society's behavior, contributing to the globalization of knowledge. In the scope of aircraft maintenance, the use of such technologies in the production processes management gains new shapes through the use of technologies capable of providing greater control of resources, ensuring the reliability of the services provided and the control of operating expenses. The main objective of this paper is to analyze the potential of using a messaging application in the management of calls in an aircraft maintenance company. Method: the methodological procedure is characterized by action research, with a focus on information and communication technologies applied in processes management. What favors the development of an efficient management model in the distribution of resources used in the tasks attendance activities. Results: the results describe the relevant use of the messaging application by Quality Control. It establishes a standard in meeting demands in aircraft maintenance context, providing improvements and contributing to a better visibility in the details of tasks and, a more efficient management of the resources used in maintenance requests. Conclusions: it ensures that the application of such technologies provides greater synergy between the various sectors involved in the process, improving communication and the precise targeting of resources to be applied.
\end{abstract}

Keywords: Information and communication technologies; Aircraft maintenance; Processes management; Message applications.

\section{INTRODUÇÃO}

A partir do desenvolvimento das tecnologias de informação, um dos principais objetivos da área da Ciência da Informação, enquanto Ciência que se dedica ao estudo de novas formas de representação dos mais variados tipos de recursos informacionais, é proporcionar subsídios teóricos acerca das tecnologias emergentes no âmbito da representação e recuperação, favorecendo a criação de ferramentas e métodos eficazes no compartilhamento informacional contemporâneo, sobretudo em ambientes dinâmicos como a Web.

Nesse contexto, a utilização de Tecnologias de Informação e Comunicação (TICs) em ambientes corporativos possibilita novas perspectivas no âmbito da gestão de processos, favorecendo a transmissão de dados em ambientes virtuais de forma massiva e favorecendo uma recuperação e compartilhamento de conteúdo de maneira mais eficiente.

A acessibilidade de conteúdos e troca de informações favorecida por aplicativos de mensagens como WhatsApp e Telegram possibilita que tais aplicativos assumam um importante papel de comunicação em ambientes corporativos, podendo ser utilizados como ferramentas para viabilizar uma comunicação direta e sinérgica, permitindo a manipulação e a reorganização de demandas nos mais diversos setores. 
No âmbito aeronáutico, contexto sobre o qual esta pesquisa versa, o uso dessas tecnologias permite uma melhor gestão dos processos inerentes à manutenção de aeronaves, uma vez que a ala competitividade desse setor requer que as empresas adotem planos estratégicos e eficientes no atendimento de suas demandas (Cruz, 2016).

Tais tecnologias possibilitam soluções técnicas e administrativas práticas, permitindo identificar pontos de melhorias nos processos de manutenção de aeronaves, visando que as metas e os objetivos de produtividade e competitividade planejados pelas empresas sejam alcançados de forma efetiva a partir da utilização de ferramentas tecnológicas já consolidadas na gestão e controle dos processos de manutenção.

Nessa perspectiva, o objetivo central dessa pesquisa consiste em analisar as potencialidades da utilização de um aplicativo de mensagens na gestão de atendimentos em uma empresa de manutenção de aeronaves.

\section{REVISÃO DE LITERATURA}

Com a globalização da informação, comunicação e, sobretudo, dos processos de produção intelectual devido aos avanços das tecnologias de informação, há o surgimento de novos paradigmas no campo da Ciência da Informação que, graças ao desenvolvimento científico das últimas décadas e novos aportes tecnológicos, tornam os processos produtivos mais eficientes ao proporcionarem o intercâmbio e interação entre as mais distintas áreas do conhecimento humano.

Tais transformações convergem para um novo cenário, no qual a comunicação e o conhecimento ocorrem em uma velocidade sem precedente na história, favorecendo o desenvolvimento de novas tecnologias estruturadas a partir de "uma grande variedade de conceitos e construtos teóricos, empíricos e pragmáticos, bem como numerosas realizações práticas" para esse processo (Saracevic, 1996, p. 44). Dito isso, Martínez-Silveira e Oddone (2007) consideram que a globalização do conhecimento tem papel fundamental na transformação cultural na chamada sociedade da informação uma vez que, dado o volume de informações, um novo paradigma vem-se firmando, relacionado a uma perspectiva agora mais socializante cuja ocorrência se dá tanto no âmbito dos sistemas informacionais quanto no dos usuários, que estão inseridos em contextos históricos e sociais que influem de modo decisivo na definição de suas características.

No entanto, duas décadas após a popularização da Web, novas tecnologias foram desenvolvidas e atualmente a rede global de computadores não se limita à disponibilização de documentos, possibilitando representar praticamente qualquer entidade do mundo real que possa ser associada a um identificador, adotando tecnologias com grande capacidade de compartilhamento de informações na Internet.

Do ponto de vista prático, as tecnologias desenvolvidas na tendência de dados interligados na Internet tornam o acesso a qualquer tipo de recurso um processo ágil no qual as informações armazenadas em bibliotecas ou banco de dados podem ser recuperadas e compartilhadas em larga escala (Silva, 2013), proporcionando ao usuário de sistemas informatizados maior autonomia em suas escolhas.

\section{AS TECNOLOGIAS DE INFORMAÇÃO E COMUNICAÇÃO}

O vertiginoso desenvolvimento da Ciência da Computação, com a criação de novas ferramentas tecnológicas, foi fator determinante para proporcionar à Ciência da Informação o desenvolvimento de métodos e modelos de representação, transmissão, transformação e reuso da informação em sistemas operacionais.

Para Ramalho (2016), o ambiente Web constitui-se como uma das principais e mais ricas fontes de informações no contexto contemporâneo, contudo, apesar de toda inovação tecnológica, os processos de recuperação da informação ainda são insuficientes diante do grande volume de conteúdo disponibilizado, seja no ambiente físico ou digital.

Nesse contexto, sob a influência de novas tendências de pesquisa envolvendo Linked Data, Data Science e Web Semântica que, em essência, possibilita a interligação dos recursos informacionais e agregação semântica em níveis mais satisfatórios, a crescente demanda por tecnologias que atendam os anseios por ferramentas capazes de trabalhar com um grande volume de dados dentro do contexto de Big Data (Souza, Martins, \& Ramalho, 2018) são cada vez mais necessárias para dar suporte a uma crescente demanda pelo controle de dados e informações de forma mais eficaz.

As TICs surgem em um cenário de grandes transformações sociais e tecnológicas no qual a velocidade no compartilhamento de conteúdos de forma instantânea está presente em praticamente todos os tipos de recursos tecnológicos disponíveis ao indivíduo na contemporaneidade, sendo elas empregadas para os mais diversos fins (Feijó, Silva, \& Benetti, 2014). Seja para uma simples comunicação em grupo, no compartilhamento de informações e dados, seja para as atividades que demandam um controle de fluxo mais eficiente de atividades produtivas.

É por meio dessas tecnologias que os aplicativos de mensagens amplamente utilizados pelo público em geral na Internet podem potencializar um grande fluxo informacional promovendo uma interação cada vez maior entre 
grupos e setores distintos em uma sociedade integrada pelas TICs, permitido que informações e dados sejam amplamente manipulados.

Com o avanço no desenvolvimento dessas tecnologias, outras ferramentas inseridas no bojo das TICs podem ser utilizadas para proporcionar uma relação mais estreita entre os setores produtivos das organizações, favorecendo a criação de oportunidades e o crescimento em seu escopo de atuação e competitividade, já que utiliza ferramentas que permitem a busca constante por melhoria contínua em seus processos, redução de custos e na melhoria da comunicação entre os diversos setores envolvidos no processo (Fagundes, 2019).

Essa nova frente de atuação demanda das organizações a capacidade de manterem-se informadas e, ao mesmo tempo, estabelecerem estratégias de comunicação eficazes com diferentes atores: o público para o qual dirigem suas atividades, seus parceiros, o poder público e, cada vez mais, dirigem-se também à sociedade como um todo, buscando apoio e visibilidade (Borges, Lessa, \& Oliveira, 2014, p. 184).

Canaday (2018) destaca que no âmbito produtivo é cada vez mais comum o uso de ferramentas tecnológicas desenvolvidas no contexto das TICs, na qual novas funcionalidades são incorporadas a partir dos avanços da Inteligência Artificial (IA), permitindo o controle de processos de forma mais assertiva e possibilitando, inclusive, a correção de possíveis falhas na execução de processos em andamento, ou prevendo e planejando um possível evento de manutenção não programado.

O emprego de tais tecnologias na gestão de processos é uma realidade cada vez mais presente em setores nos quais as tomadas de decisão necessitam ocorrer de forma ágil, sem perda do foco e das diretrizes pré-determinadas no projeto inicial. Associadas às demais práticas do sistema produtivo, essas tecnologias contribuem de forma significativa para o redesenho na forma de atendimento, minimizando perdas e potencializando oportunidade de ganhos em setores de apoio que são determinantes para o fluxo sistêmico no âmbito da gestão.

\section{A GESTÃO DE PROCESSOS NA MANUTENÇÃO DE AERONAVES}

Estabelecer metas, traçar estratégias e estipular prazos é essencial para o sucesso de qualquer projeto. No setor aeronáutico, tal máxima não poderia ser melhor definida, uma vez que uma aeronave parada em solo representa prejuízo. Segundo Correia (2012) a gestão da manutenção é um ponto importante que vem ganhando relevância significativa ao longo dos anos, uma vez que os recursos e os custos empregados nesse segmento podem ser dimensionados de forma mais eficiente pelas empresas.

A eficácia na gestão da manutenção requer uma boa sinergia com as demais áreas que dão suporte a esse setor, já que o gerenciamento de informações e o planejamento estratégico entre as equipes se tornam essenciais para o sucesso do projeto como um todo (Machado, Urbina, \& Eller, 2015).

Dessa forma, uma boa interface entre os diversos setores que atuam diretamente no processo de manutenção se dá a partir de um planejamento estratégico eficiente, que consiste em uma estruturação do sistema de inteligência competitiva da organização no qual o elemento-chave é a criação de um fluxo constante de informação que possibilita a tomada de decisão correta e monitoramento sistemático dos recursos disponíveis.

Segundo Cruz (2016), a gestão dos processos de manutenção deve atender a decisões estratégicas da organização, nas quais o cumprimento de prazos, a qualidade dos serviços e a capacidade de resiliência diante das mudanças ao longo do projeto devem obedecer a um único propósito cujas metas estabelecidas devem ser claras e os resultados alcançados em sua totalidade por todo o grupo envolvido.

Administrar de forma eficiente todos os objetivos propostos, transformando-os em ações efetivas na organização, é uma missão essencial para garantir que o planejamento estratégico, a organização e o controle de todos os esforços realizados nos processos de manutenção atinjam seus objetivos primários de maneira satisfatória, garantindo a competitividade, a qualidade e o uso adequado dos recursos empregados (Chiavenato, 2003; Correia, 2012).

Nesse cenário, as organizações têm à sua disposição uma gama de tecnologias capazes de desempenhar um controle eficaz dos recursos a serem empregados na manutenção, permitindo uma melhor interligação entre áreas, compartilhamento de informações importantes para o bom andamento do projeto e a possibilidade de definir quais as práticas mais adequadas devem ser adotadas.

Com isso, surge uma nova tendência de uso das TICs nesse setor, no qual novas formas de compartilhamento de informações permitem gerenciar recursos e tempo empregados nas atividades de manutenção, de forma que todos os profissionais da organização compartilhem o mesmo objetivo, influenciando a produtividade e os bons resultados, cujo objetivo final é a utilização das melhores práticas para se alcançar o estado de excelência.

\section{O USO DAS TICS NA GESTÃO DE PROCESSOS NA MANUTENÇÃO}

O desenvolvimento e a aplicação das TICs como ferramentas no âmbito da gestão de recursos acarretam mudanças significativas nos processos produtivos da indústria contemporânea, proporcionam resultados mais eficientes 
quando empregados em conjunto com práticas profissionais consolidadas e contribuem para a melhoria dos processos, evitando retrabalhos e perdas de recursos no decorrer do projeto.

A qualidade na fabricação de produtos e na prestação de serviços ganha força a partir da utilização das TICs, empregadas para gerenciar os mais diversos processos na cadeia produtiva e sistematizar de forma eficiente o uso dos recursos disponíveis, evitando os custos elevadíssimos resultantes dos erros decorrentes de manipulações e interpretações equivocadas de informações essenciais ao processo.

Albrecht (2001) destaca que a qualidade das informações para a gestão dos processos na manutenção é fundamental para as tomadas de decisão no contexto produtivo, pois auxiliam os gestores na avaliação de problemas e oportunidades, na priorização da mudança de atividades e no redesenho de sistemas, processos e práticas.

Tal percepção é ratificada por R. R. Silveira, Ottani, Varvakis, Bastos, e Franzoni (2016) que destacam que, com a difusão e uso das TICs nesse contexto, torna-se possível a integração de diferentes áreas, a circulação e troca de dados, a disseminação de informações nas redes eletrônicas e a geração do conhecimento.

O uso de tais tecnologias possibilita uma redução significativa nos custos operacionais de uma organização, uma vez que o emprego de tempo, recursos humanos, financeiros, dentre outros podem ser dimensionados para um melhor aproveitamento dos mais diversos recursos que são destinados ao controle e coordenação da manutenção, tornando todos os processos mais ágeis e integrados entre si (Machado et al., 2015).

Uma das tecnologias emergentes utilizadas para a comunicação de maneira instantânea é o aplicativo Telegram que, nesta pesquisa, é abordado pela sua aplicação no controle e gestão de recurso humano na área do Controle da Qualidade (CQ) de uma unidade de Maintenance, Repair and Overhaul (MRO), ou centro de manutenção de aeronaves e componentes de uma empresa aérea, que o utiliza na gestão e controle de atendimento de tarefas de manutenção.

A proposta surge da necessidade de se estabelecer um padrão no acionamento e reduzir o tempo de espera no atendimento de tarefas de manutenção classificadas como Required Inspection Item (RII) ou Itens de Inspeção Requerida (Graciano, 2014), que demandam acompanhamento de um inspetor do Controle da Qualidade. Tal atividade compreende uma alta demanda por parte do setor que se depara com um grande volume de acionamentos e uma demora considerável para o atendimento devido ao deslocamento, em muitos casos, desnecessários entre os hangares de manutenção.

Antes da implementação da ferramenta como dispositivo de atendimento, o acionamento dos inspetores dava-se por meio da solicitação via líder do departamento do CQ que, dependendo da disponibilidade de sua equipe, acionava um inspetor específico da área para o atendimento requerido. A grande problemática nesse processo estava no tempo de espera, pela manutenção, que girava em torno de 30 a 40 minutos para que um inspetor fosse acionado.

Outros fatores contribuintes para o tempo elevado no atendimento eram a falta de critério na priorização das tarefas, a falta de padronização no processo de acionamento, a aeronave não ser configurada para a realização das inspeções e a não visualização, pelo controle da qualidade, das demandas críticas (tarefas que devem ser realizadas em um período determinado do check).

O método de Cronoanálise (Aguiar, 2006) foi utilizado para a coleta de dados e o levantamento do tempo gasto no processo entre o acionamento pela manutenção até o pronto atendimento pela equipe do CQ, no qual foi detectado um tempo médio de aproximadamente 30 minutos, levando-se em consideração as variáveis inerentes ao processo, quantidade de acionamentos entre as diversas aeronaves em check, chamados realizados de forma equivocada, distância entre os hangares com maior número de acionamentos, dentre outros fatores.

A adoção do modelo tradicional de atendimento, até então praticado pela equipe do Controle da Qualidade, gerava para a área de manutenção um Not Value Aggregate (NVA) (Ishikawa, 1985), Valor Não Agregado, de 5 $\mathrm{min} /$ tec/dia ao longo dos turnos de trabalho representando $21 \%$ do total do NVA, dependendo da quantidade de tarefas que eram classificadas RII e o tipo de check em questão. Neste mesmo comparativo, foram utilizados os dados relativos a outras áreas de apoio que foram incluídas no projeto original, mas que não fazem parte desse objeto de estudo.

Neste estudo foram considerados dados coletados e aplicáveis às três linhas e turnos de trabalho existentes no MRO durante o período de 12 dias. Foram coletados 1249 acionamentos, com $7 \%$ desse total cancelados, sendo $87 \%$ (1088) chamados direcionados à equipe do CQ e 13\% (161) direcionados à equipe de Non Destructive Test (NDT), que está subordinada à gerência de Qualidade, mas que não entra nessa pesquisa.

Testes iniciais demonstraram de pronto a eficiência da proposta que, a partir da integração do bot do aplicativo com o sistema MROSYs (Graciano, 2014), que gerencia os apontamentos de entrada e saída de mecânicos e inspetores do Controle da Qualidade nas tarefas de manutenção, o disparo das solicitações para o atendimento de tarefas RII seriam feitos de forma automática para todos os membros do grupo que tivessem realizado seu login de acesso ao programa incorporado no aplicativo. 
A implementação e integração entre os sistemas usados nesse projeto melhorou significativamente o tempo de atendimento, levando $85 \%$ dos acionamentos para um tempo de espera menor do que 30 minutos com uma meta inicial agressiva de 90\%. O tempo médio de atendimento teve uma média geral de 17 minutos e, considerando os 90\% melhores tempos, a média foi de 10 minutos, conforme demonstrado na Figura 1.

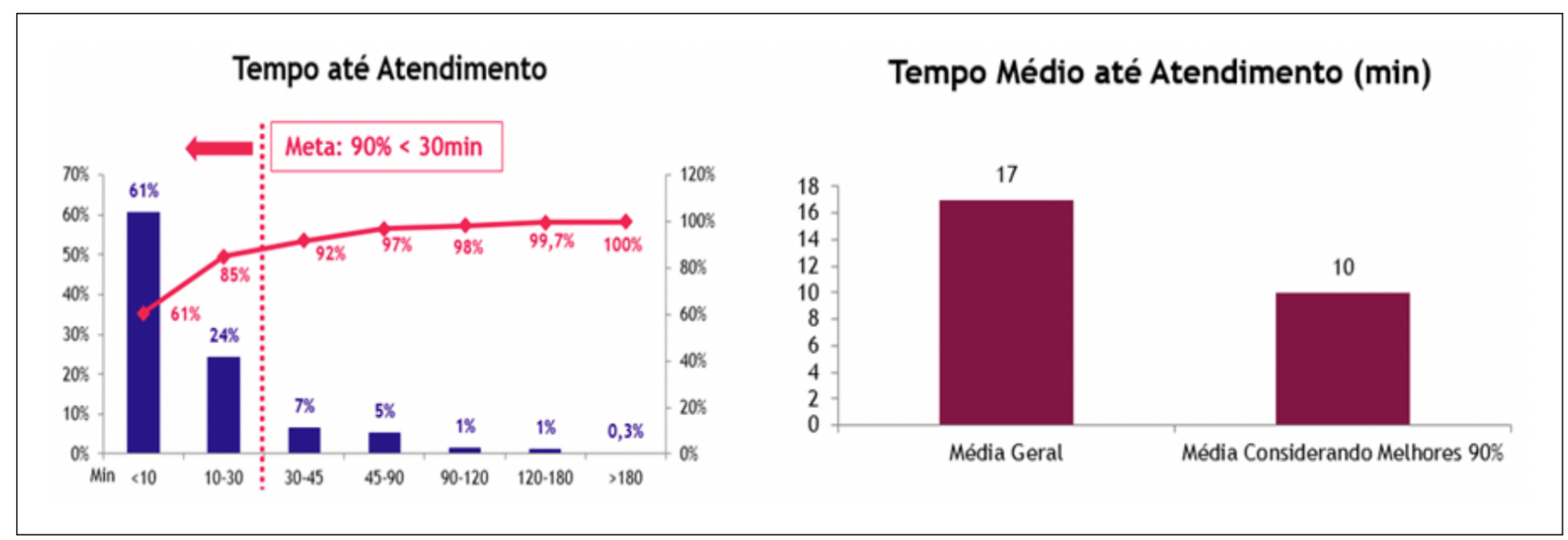

Figura 1. Levantamento médio de atendimento

Fonte: Martins, Ramalho, e dos Santos Graciano (2020).

Após a realização de vários testes ao longo dos checks, que se sucederam na manutenção pelo período de um mês, realizados nas três linhas e nos três turnos de operação, estipulou-se que o tempo médio de atendimento seria em torno de 10 minutos considerando que os requisitos preestabelecidos no projeto inicial sejam cumpridos para que não haja a incidência de retrabalho nas configurações prévias da tarefa.

Mostrando-se um modelo eficiente no planejamento de recursos para essa atividade, dado a redução significativa de tempo nos atendimentos solicitados que saíram de valor médio de 30 para 10 minutos como destacado na Figura 1, o modelo proposto acabou sendo implementado de forma permanente, sendo adicionadas ao aplicativo novas funcionalidades que contribuíram para um ganho expressivo na quantidade de tarefas atendidas por linha, pelo Controle da Qualidade.

A utilização do aplicativo Telegram estabeleceu um padrão nos atendimentos do Controle da Qualidade diante das demandas provenientes da manutenção, contribuindo para uma melhor visibilidade no detalhamento das tarefas classificadas como RII (como setup, execução e close-up das tarefas) e uma gestão mais eficiente dos recursos empregados, uma vez que tarefas consideradas críticas têm atendimento prioritário, pois o atraso em sua conclusão pode impactar negativamente no cumprimento das demais. Ademais, estabeleceu-se uma padronização nos critérios de priorização de atendimento, possibilitando verificar a real necessidade de envolvimento dos inspetores nos diversos tipos de solicitações realizadas pela manutenção.

Como resultado desse processo, é apresentado na Figura 2 o fluxograma da nova tratativa de acionamento implementado nas áreas do CQ e NDT, ambos sob responsabilidade da Gerência de Qualidade, que passaram a adotar esse novo formato observando os pré-requisitos necessários para o acionamento, possibilitando um melhor direcionamento de pessoal, planejamento diário das tarefas de manutenção e adequação das demandas para as áreas tanto do CQ, quanto da manutenção.

Com essa nova estrutura de acionamento, quando verificada a necessidade de atendimento, a manutenção faz a solicitação via sistema que, imediatamente, apresenta a notificação no aplicativo que, automaticamente, começa a contabilizar o tempo definido para o atendimento. Transcorridos os 10 minutos necessários para o Service Level Agreement (SLA) ou Tempo Limite para o atendimento, e não havendo nenhum contato por parte do CQ, o líder da manutenção será acionado pelo técnico que fez a solicitação e determinará se a tarefa é ou não de caráter crítico (atendimento imediato). Caso seja determinado como tarefa crítica (que compromete o andamento das demais tarefas), o atendimento passa a ter prioridade sobre as demais tarefas selecionadas para área ou para a linha de manutenção como um todo.

O fator positivo no modelo implementado está exatamente na forma de gestão em que as equipes tanto do CQ, quanto da manutenção, desempenham ao terem ao seu dispor uma maneira eficiente de planejamento e adequação às demandas oriundas do processo de manutenção. Com isso, as tarefas consideradas críticas, ou seja, caso não sejam cumpridas no prazo determinado podem impactar de forma negativa o andamento do check, podem ter seu atendimento priorizado uma vez que há uma maior sinergia entre as equipes de manutenção e de apoio, o Controle da Qualidade. 


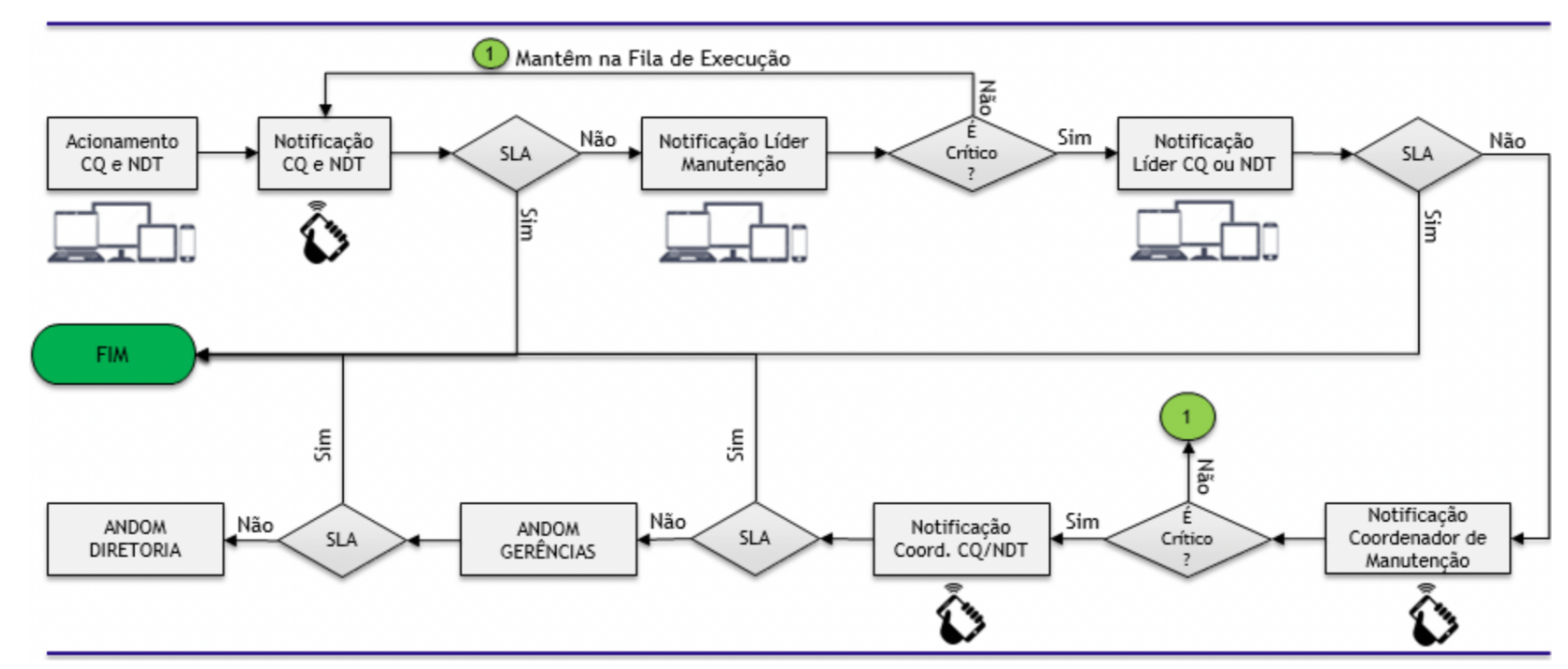

Figura 2. Fluxo de acionamentos Fonte: Martins et al. (2020).

\section{PROCEDIMENTOS METODOLÓGICOS}

Quanto aos procedimentos metodológicos, utilizou-se o método de pesquisa-ação, uma vez que se objetiva identificar os principais fatores relacionados a um determinado problema que, neste estudo, tem foco na temática de TICs aplicadas na gestão de processos no setor de manutenção aeronáutica que auxilia no desenvolvimento de diretrizes e soluções práticas a serem empregados para a melhoria contínua na gestão de processos (McKay \& Marshall, 2001).

Segundo Thiollent (2005) a metodologia de pesquisa-ação favorece a identificação das problemáticas inerentes ao contexto abordado e contribui para o desenvolvimento de ações efetivas e duradouras. Caracteriza-se, também, como pesquisa de natureza aplicada, uma vez que "objetiva gerar conhecimentos para aplicação prática, dirigidos à solução de problemas específicos" (D. T. Silveira \& Coórdova, 2009, p. 35)

Abordam-se os aspectos de utilização das TICs no âmbito de prestação de serviços, como o setor de manutenção de aeronaves que, dado o grande volume de informações, utiliza as TICs para auxiliar as tomadas de decisão e gestão de seus processos. Destaca-se o emprego dessas tecnologias nesse setor e sua potencial contribuição para a padronização dos atendimentos prestados pelo Controle da Qualidade, que passa a planejar e controlar de forma eficiente o emprego de seus recursos, favorecendo uma melhor sinergia entre diversas áreas envolvidas nesse processo.

\section{CONSIDERAÇÕES FINAIS}

O desenvolvimento de novas tecnologias presenciado nos últimos anos representa um grande avanço no setor produtivo ao dispor ferramentas capazes de proporcionar um controle mais efetivo dos processos de manutenção ou manufatura de partes. No setor aeronáutico, o emprego de tais tecnologias pode representar um grande passo na gestão de processos ao possibilitar o uso de informações precisas e seletivas para as tomadas de decisão e promover uma melhor sinergia entre os diversos setores envolvidos diretamente ou não no processo.

Planejar de forma eficiente os recursos empregados na manutenção de aeronaves é essencial para a redução de gastos e de retrabalhos nas atividades inerentes a esse setor, uma vez que estabelece metas e diretrizes para garantir a qualidade, confiabilidade e entrega, no prazo, de produtos e serviços.

A utilização do aplicativo Telegram no controle dos atendimentos prestados pela equipe do Controle da Qualidade mostrou-se eficaz ao proporcionar uma interação sincrônica entre as linhas de manutenção existentes no MRO e o CQ, permitindo um ganho significativo nos atendimentos ao reduzir de 30 para 10 minutos o tempo máximo de espera, e na qualidade dos serviços prestados ao estabelecer um padrão no atendimento das demandas provenientes da manutenção, contribuindo para uma melhor visibilidade no detalhamento das tarefas e uma gestão mais eficiente dos recursos empregados uma vez que, pode-se verificar a real necessidade de envolvimento dos inspetores nos diversos tipos de solicitações realizadas pela manutenção.

Cada vez mais recursos são implementados na ferramenta, visando ampliar o escopo de atendimento da equipe do Controle da Qualidade e tornando o atendimento e demais demandas atribuídas à equipe mais ágeis e 
sincronizadas com as propostas de segurança, qualidade, confiabilidade e entrega inerentes ao processo de manutenção de aeronaves.

Nesse sentido, a proposta deste estudo é apresentar um compêndio do emprego de TICs, abordando neste artigo o Telegram, tecnologias essas empregadas no contexto das novas ferramentas tecnológicas desenvolvidas para a chamada quarta revolução industrial, que representa uma nova tendência no setor produtivo e de manutenção ao empregar as TICs na gestão de seus processos.

Acredita-se que, com o emprego cada vez maior dessas tecnologias em segmentos produtivos e de manutenção, o índice de aproveitamento de recursos, seja humano ou não, propicia uma melhor gestão, uma vez que saber-se-á realizar um melhor direcionamento e controle das atividades planejadas ou não, reduzindo com isso o tempo de respostas para os desvios encontrados ao longo do processo. 


\section{REFERÊNCIAS}

Aguiar, S. (2006). Integração das ferramentas da qualidade ao pdca e ao programa seis sigma. Nova Lima: INDG.

Albrecht, K. (2001). A Terceira Revolução da Qualidade. Knowledge Management Press and Consulting.

Borges, J., Lessa, B., \& Oliveira, L. (2014). O papel dos sites de redes sociais nas estratégias comunicativas de organizações da sociedade civil de salvador-bahia-brasil. OBS Journal, 8(3), 183-203. Recuperado de http:/ www.scielo.mec.pt/scielo.php?script=sci_arttext\&pid= S1646-59542014000300010\&lng=pt\&nrm=iso

Canaday, H. (2018). The future of MRO is human-machine teaming. Recuperado de https://www.mro-network.com/technology/future-mro -human-machine-teaming?NL $=\mathrm{AW}$-022\&Issue $=\mathrm{AW}$ -022 20180924 AW-022 776\&sfvc4enews $=42 \& \mathrm{cl}=$ article 1\&utm rid=CPĒ - E1000003186818\&utm campaign $=16551 \&$ utm medium $=$ email\&elq $2=$

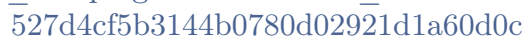

Chiavenato, I. (2003). Introdução à teoria geral da administração. Rio de Janeiro: Elsevier.

Correia, V. M. M. (2012). O programa de manutenção de aeronaves e a sua importância na gestão da continuidade da aeronavegabilidade (Dissertação de mestrado). Instituto Superior Técnico Lisboa, Lisboa.

Cruz, P. R. F. d. (2016). Qualidade na indústria aeronáutica: aplicação da ferramenta FMEA em um setor de montagem de componentes de motores aeronáuticos. In Xii congresso nacional de excelência em gestão e iii inovarse, 2016. Rio de Janeiro. Recuperado de http://www.inovarse.org/node/29

Fagundes, E. (2019). Contribuições de TIC para a Estratégia de Negécios. Recuperado de https://efagundes.com/artigos/ contribuicoes-de-tic-para-a-estrategia-de-negocios /

Feijó, L. P., Silva, N. B., \& Benetti, S. P. d. C. (2014). Impacto das Tecnologias de Informação e Comunicação na Técnica Psicoterápica Psicanalítica. Trends in Psychology, 26(3), 1633-1647. Recuperado de http://www.scielo.br/scielo.php?script=sci_arttext\&pid $=$ $\mathrm{S} 2358-18832018000301633 \& \operatorname{lng}=\mathrm{en} \& \mathrm{nrm}=$ iso

Graciano, H. L. d. S. (2014). Aplicação de uma ferramenta de business intelligence para análise das causas de variabilidade de mão de obra no contexto de manutenção de aeronaves (Trabalho de Conclusão de Curso). Universidade Federal de São Carlos, São Carlos.

Ishikawa, K. (1985). What is total quality control? the japanese way. Englewood Cliffs: Prentice-Hall. (Trad. de David Lu)

Machado, M. C., Urbina, L. M. S., \& Eller, M. A. G. (2015). Manutenção aeronáutica no brasil: distribuição geográfica e técnica. Gestão \& Produção, 22(2), 243-253. Recuperado de http://www.scielo.br/scielo.php?script=sci_arttext\&pid= S0104-530X2015000200243\&lng=en\&nrm=iso.

Martínez-Silveira, M., \& Oddone, N. (2007). Necessidades e comportamento informacional: conceituação e modelos. Ciência da Informação, 36(2), 118-127. Recuperado de http://www.scielo.br/scielo.php?pid=S0100 $-19652007000200012 \&$ script=sci_abstract\&tlng $=$ pt

Martins, P. G. M., Ramalho, R. A. S., \& dos Santos Graciano, H. L. (2020). Análise do uso do aplicativo Telegram para o controle de processos de manutenção de aeronaves. Informação É Informação, 25(1), 171-188. Recuperado de http://www.uel.br/revistas/uel/index.php/informacao/ article/view/38129

McKay, J., \& Marshall, P. (2001). The dual imperatives of action research. Information Technology \& People, 14(1), 46-59. Recuperado de http://dx.doi.org/10.1108/ 09593840110384771

Ramalho, R. A. S. (2016). Bibframe: modelo de dados interligados para bibliotecas. Informação \& Informação, 21(2), 292-306. Recuperado de http://doi.org/ 10.5433/1981-8920.2016v21n2p292 doi: 10.5433/19818920.2016v21n2p292

Saracevic, T. (1996). Ciência da informação: origem, evolução e relações. Perspectivas em Ciência da Informação, 1(1), 41-62. Recuperado de http://www.brapci.inf.br/v/a/3224

Silva, R. E. d. (2013). As tecnologias da Web Semântica no domínio bibliográfico (Dissertação de mestrado, Universidade Estadual Paulista Júlio de Mesquita Filho, Marília). Recuperado de http://hdl.handle.net/11449/93653

Silveira, D. T., \& Coórdova, F. P. (2009). Origens e evolução da Ciência da Informação. In T. E. Gerhardt \& D. T. Silveira (Eds.), Métodos de pesquisa. Porto Alegre: Editora da UFRGS.

Silveira, R. R., Ottani, N., Varvakis, G., Bastos, R. C., \& Franzoni, A. M. B. (2016). O uso da tecnologia de informação e comunicação como recurso na elaboração estratégica da informação: um estudo de caso na Gol Transportes Aéreos Ltda. In 13 simpésio de engenharia de produção. Bauru. Recuperado de http://www.simpep.feb.unesp.br/ anais_simpep_aux.php? $\mathrm{e}=13$

Souza, J. L., Martins, P. G. M., \& Ramalho, R. A. S. (2018). Modelos de reprensentação semântica na era do big data. Brazilian Journal of Information Science, 12(3), 3440. Recuperado de https://doi.org/10.36311/1981-1640.2018 .v12n3.04.p34 doi: 10.36311/1981-1640.2018.v12n3.04.p34

Thiollent, M. (2005). Metodologia da pesquisa-ação. São Paulo: Cortez Editora.

Martins, P. G. M., Ramalho, R. A. S. \& Lopes, D. C. F. (2019). Tecnologias da Informação: a gestão de processos através de aplicativos de mensagem. AtoZ: novas práticas em informação e conhecimento, 8(2), 83 - 90. Recuperado de: http://dx.doi.org/10.5380/ atoz.v8i2.71368 